\title{
Chaotic Vibration Analysis of the Bottom Rotating Drill String
}

\author{
Qilong Xue, ${ }^{1,2}$ Ruihe Wang, ${ }^{1}$ Feng Sun, ${ }^{3}$ and Zhiyuan Huang ${ }^{3}$ \\ ${ }^{1}$ College of Petroleum Engineering, China University of Petroleum, Qingdao 266580, China \\ ${ }^{2}$ Department of Electrical and Computer Engineering, University of Calgary, Calgary, AB, Canada T2N 1N4 \\ ${ }^{3}$ Drilling Technology Research Institute, Sinopec Shengli Oil field Administration, Dongying 257000, China \\ Correspondence should be addressed to Qilong Xue; xqlfly@gmail.com
}

Received 13 August 2013; Accepted 23 January 2014; Published 27 February 2014

Academic Editor: Nuno Maia

Copyright (C) 2014 Qilong Xue et al. This is an open access article distributed under the Creative Commons Attribution License, which permits unrestricted use, distribution, and reproduction in any medium, provided the original work is properly cited.

Drill string vibration is a widely studied topic. This paper developed a real-time measurement system near the drilling bit and extracted the lateral vibration, longitudinal vibration time series of bottom rotating drill string. In order to reconstruct the phase space, we estimated the delay time with mutual information and calculated the embedding dimension through Cao's method. Finally, the chaotic characterization of the system is analyzed by calculating the correlation dimension and the largest Lyapunov exponent. The results show that such system can exhibit positive finite-time Lyapunov exponents and a clear convergence toward the correlation dimension, which is a strong indicator for the chaotic behavior of the system. It is expected that the new dynamics found in this paper could be of potential implication to the control methods of the drill string vibration.

\section{Introduction}

In oil and gas drilling engineering, the well is created by drilling a hole 5 to 50 inches $(127.0 \mathrm{~mm}$ to $914.4 \mathrm{~mm})$ in diameter into the earth with a drilling rig that rotates a drill string with a bit attached. In the process, severe drill string vibration is a major contributor to downhole tool failure. It may also cause hole damage and increase the need for more frequent rig repair. Typically, the drilling string vibration can be divided into three types or modes: lateral, longitudinal, and torsional. The destructive nature of each type of vibration is different.

Lateral and longitudinal vibrations of the drill string have been undertaken extensive research since it proposed from mid-1960s, The main reason that caused the fatigue failure of the bottomhole assembly (BHA) [2] was considered to be the vibration of the drill string. Many studies of the drill string focused on the determination of natural frequencies $[3,4]$, bending stress calculation [5, 6], stability analysis [7], lateral displacement prediction [8], and so forth. Spanos et al. [9] established the finite element model of drill string lateral vibration and analyzed nonlinear random vibration.
Chunjie and Tie [10] obtained the natural frequency of drill string longitudinal vibration from a finite element model. Vibrations of all three types (lateral, longitudinal, and torsional) may occur during rotary drilling and are coupled. Single vibration model cannot well describe the dynamics of BHA; furthermore, to establish a precise bottom hole kinetic theory model is difficult to achieve because of the underground complex situation. Additionally, the process of drill bit break rocks is a nonlinear process which is difficult to predict; this will inevitably lead to the drill bit vibrations which present extremely complex dynamics [11], even the chaotic phenomena. Some researchers have established a theoretical model analysis of the drill string vibration [12] and proved that the drill string vibration could present the chaotic vibration in the drilling process. However, further proof that the deterministic chaos exists in the drilling practice is missing.

With the development of measurement while drilling (MWD) technology, in recent years the use of downhole measurement data to analyze the mechanical properties and vibration characteristics of the BHA has become the trend [13]. In particular, we could obtain the data form the near-bit 


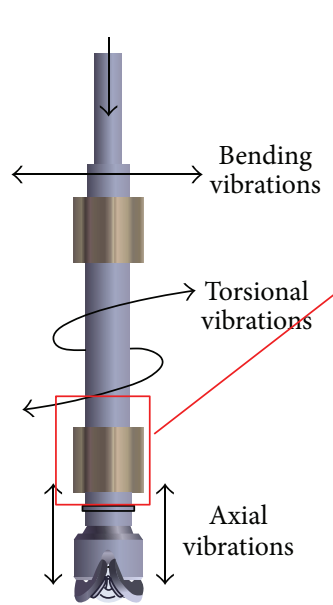

(a)

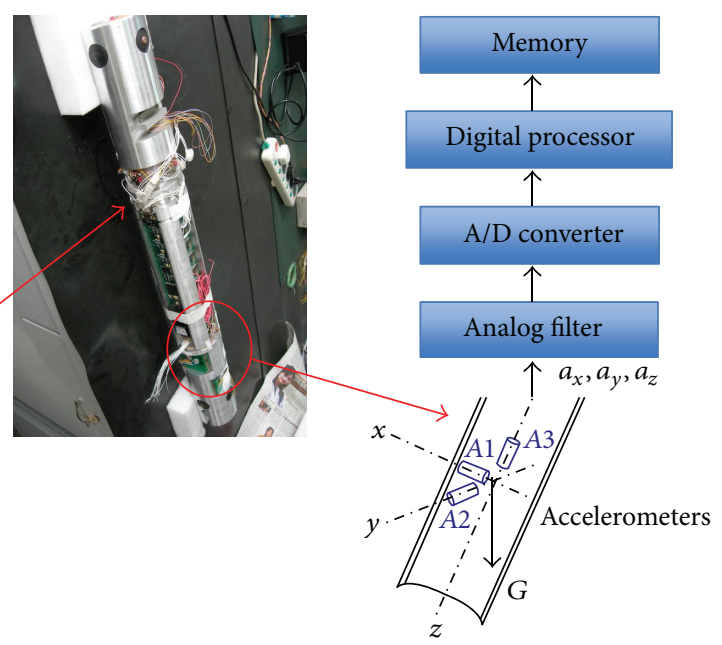

(b)

(c)

FIGURE 1: Measurement system. (Part (a) is the schematic diagram of drill string mechanism. Part (b) is the structure chart of mearsurement system, installed inside the central axis of the drill collar shown in red block. Part (c) indicates the install configuration of triaxial accelerometers; the signals will be stored in the memory chip; $\mathrm{G}$ is the acceleration of gravity.)

measurement [14], which is the technology that installs the measurement system near the drilling bit. Thus, we can analyze the drilling bit vibration that could be supported by the field data. However, there is no report describing the vibration characteristics of the drill string with the measurement time series. This paper developed a "strap down" MWD system and measured the drill bit lateral vibration and longitudinal vibration real time, the frequency up to $100 \mathrm{~Hz}$. The system stored sampling data real time in drilling process, which could be play-backed after drilling. Thus we can obtain the massive time series data of the drill bit vibration. Then we use time series analysis method to study dynamics of drill bit with measurement data. The results show that such system can exhibit positive finite-time Lyapunov exponents and a clear convergence toward the correlation dimension, which is a strong indicator for the chaotic behavior of the system.

\section{Data Acquisition}

The use of vibration measurements to detect bit and drill string dysfunctions in real time can improve drilling efficiency. We developed a real-time measurement system near the drilling bit and incorporated three-axis accelerometers arranged in three mutually orthogonal directions. Figure 1 shows the installed structure of the downhole measurement system. The system is installed inside the central axis of the drill collar and distanced from drill bit around two meters.

As shown in Figure $1, a_{x}, a_{y}$, and $a_{z}$ are, respectively, defined as survey signal of triaxial accelerometers on the $x y z$ axis; then the resultant acceleration is defined as $A=\sqrt{a_{x}^{2}+a_{y}^{2}+a_{z}^{2}}$. The sensors measurement frequency is $100 \mathrm{~Hz}$. We used $a_{h}=\sqrt{a_{x}^{2}+a_{y}^{2}}$ to express the lateral vibration of the drilling bit and used $a_{z}$ to express the longitudinal vibration of the drilling bit. The acceleration of gravity will cause the bias of the time series; thus we use empirical mode decomposition (EMD) that is a detrending method and does not distort the shape of the spikes. Figure 2 shows the time series and spectral density estimation of the lateral vibration and longitudinal vibration. The data has been extracted from the field tests.

We used Welch's method [15] approach to spectral density estimation. The method is based on the concept of using periodogram spectrum estimates, which are the result of converting a signal from the time domain to the frequency domain. Welch's method reduces noise in the estimated power spectra in exchange for reducing the frequency resolution. Due to the noise caused by imperfect and finite data, the noise reduction from Welch's method is often desired. From the spectral density, we can find that the system might to be chaotic since there are no obvious peaks.

Theoretically, it is enough for the measurement system to only have three accelerometers that were installed in the instrument coordinate system. Actually, we designed our measurement system with a redundancy scheme in order to effectively improve the system reliability. In the module design, the AD mode applies two DSP chips (TMS320F2812) as the processor, with combination of the three AD7656 chips to complete the data collection.

\section{Methodology}

In order to investigate deterministic chaos in the time series of our system, we used the phase-space reconstruction method to reconstruct an attractor that preserves the invariant characteristics of the original attractor. We estimated the correlation dimension and the largest Lyapunov exponent in order to estimate the invariants of the attractor. 


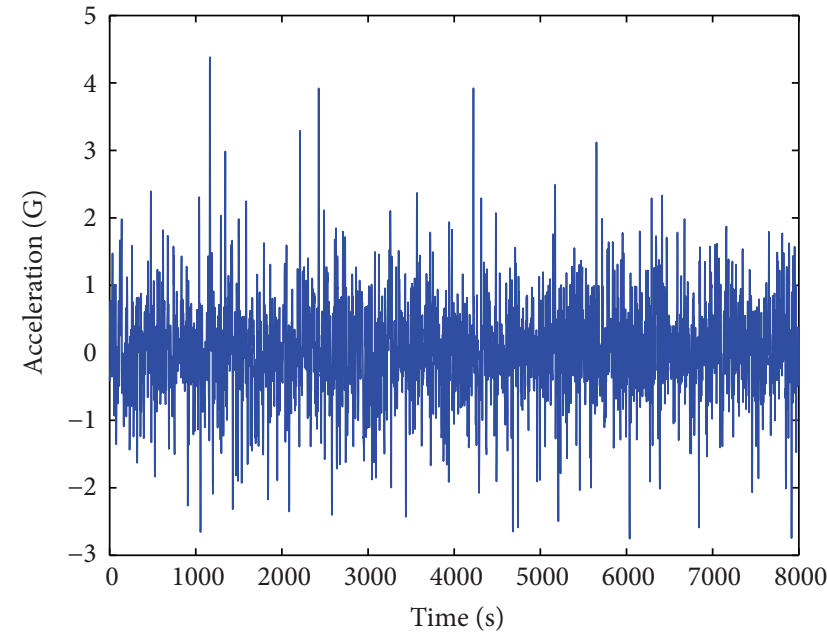

(a) Time series of $a_{h}$

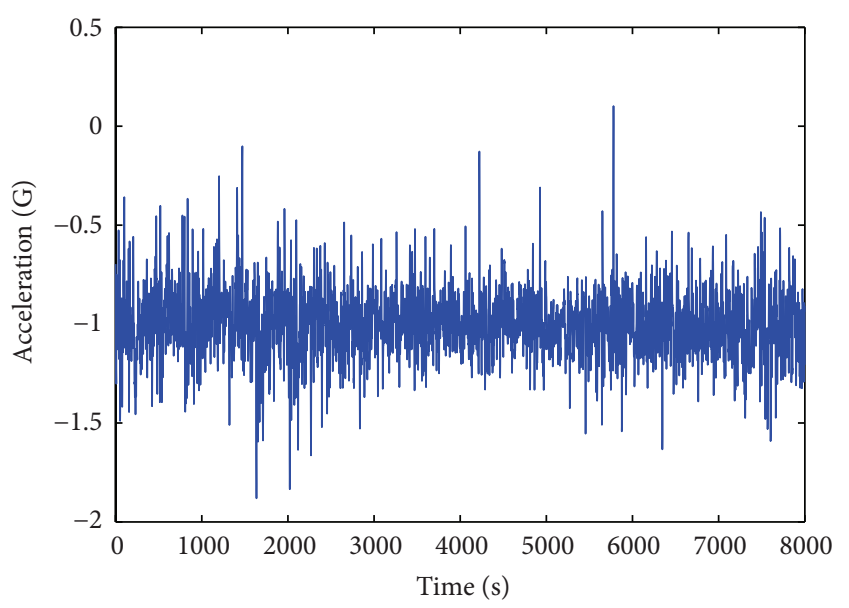

(c) Time series of $a_{z}$

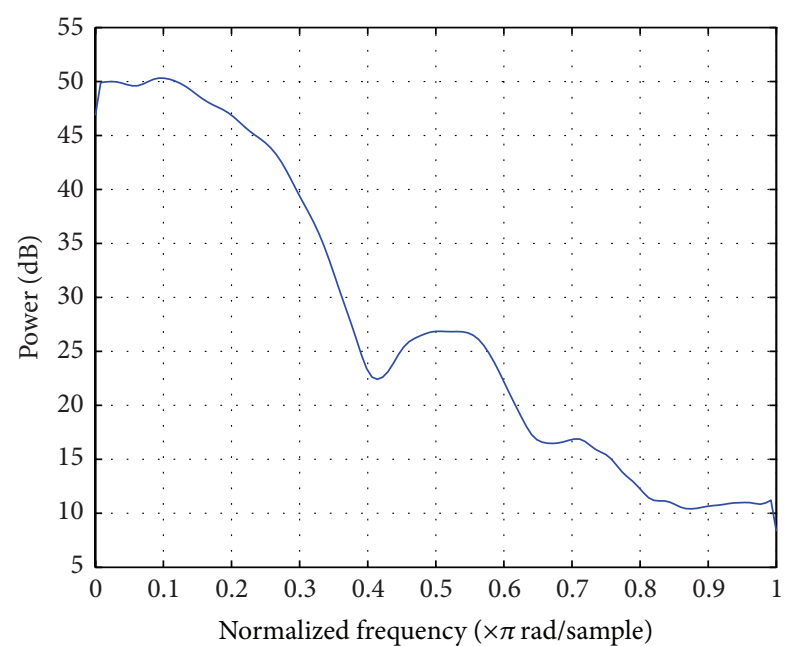

(b) Spectral density estimation of $a_{h}$

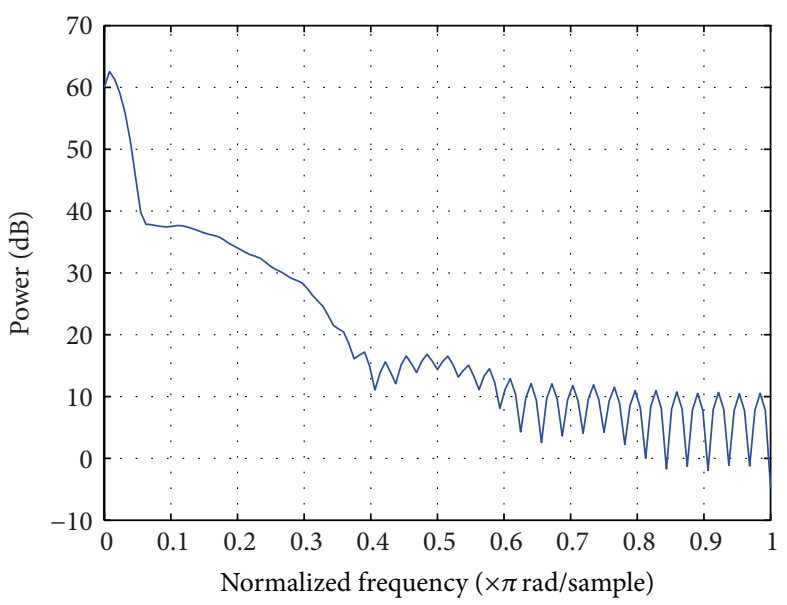

(d) Spectral density estimation of $a_{z}$

FIGURE 2: Time series and spectral density estimation (the top plane is produced from the signal of lateral vibration and the bottom plane is produced from the signal of longitudinal vibration).

3.1. Phase-Space Reconstruction. The dynamics of the time series $x_{0}, x_{1}, \ldots, x_{n-1}$ are fully captured or embedded in the $m$-dimensional phase space, $m \geq d$, where $\mathrm{d}$ is the dimension of the original attractor. A vector $\vec{x}_{i}$ in the reconstructed phase space is constructed from the time series as follows:

$$
\vec{x}_{i}=\left[x_{i}, x_{i-\tau}, \ldots, x_{i-(m-1) \tau}\right]
$$

where $\tau$ is the delay time.

We apply the mutual information [15] to estimate a proper embedding delay $\tau$. Fraser and Swinney [16] consider the use of the first minimum of the mutual information as the optimal embedding delay. Moreover, we use Cao's method [17] to determine the embedding dimension. It computes $E_{1}$ and $E_{2}$ for the data set of dimension 1 up to a dimension of $D$ that is the largest embedding dimension used for calculation. $E_{1}$ and $E_{2}$ are defined as follows:

$$
\begin{gathered}
E_{1}(d)=\frac{1}{N-d \tau}\left|\sum_{i=1}^{N-d \tau}\right| x_{i+d \tau}-x_{n(i, d)+d \tau}|| \\
E_{2}(d)=E_{1}(d+1) / E_{1}(d),
\end{gathered}
$$

wherein $d$ is the embedding dimension, $N$ is the number of data points, $\tau$ is the embedding delay, $x_{i+d \tau}$ and $x_{n(i, d)+d \tau}$ are the $i$ th vector in the data sets and its nearest neighbors of $d$ dimensional phase space [18]. $E_{1}$ is only dependent on the dimension $m$ and the delay time $\tau$. To investigate its variation from $m$ to $m+1$, we define $E_{2}$. $E_{1}$ is calculated for determining the minimum embedding dimension of time series and $E_{2}$ for distinguishing deterministic data from random data.

3.2. Correlation Dimension. After the reconstruction of time series in phase space, we can determine whether it has 


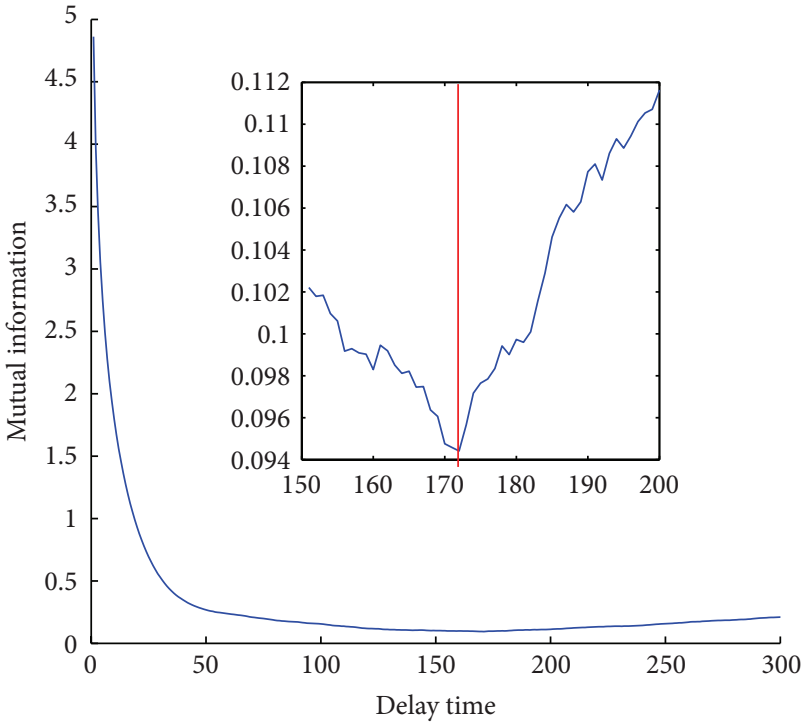

(a)

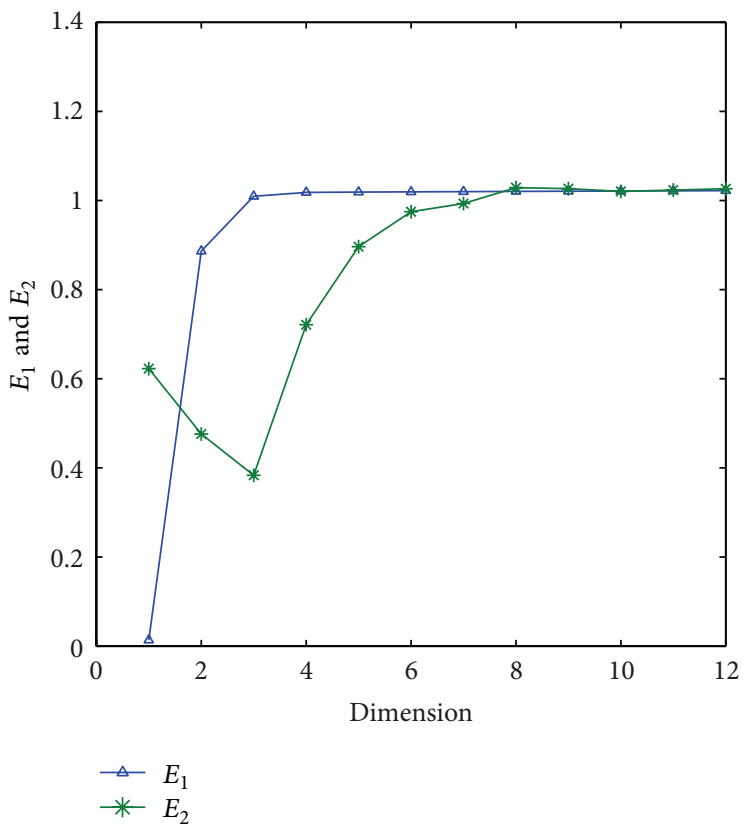

(b)

FIgURE 3: Determination of the proper delay time and embedding dimension of the lateral vibration $(\tau=172, d=8)$.

chaotic properties by calculating the dynamic invariants. Common dynamic invariants are correlation dimension [19], Kolmogorov entropy [20], the Lyapunov exponent [21], and so on. The correlation dimension method can be used for detecting the present possibility of chaos. An algorithm proposed by Grassberger and Procaccia (GP) [19] is the most commonly applied method. According to this method, the correlation sum $C(r)$ is expressed as

$$
C(r)=\frac{2}{N(N-1)} \sum_{i=1}^{N} \sum_{j=i+1}^{N} H\left(r-\left\|x_{i}-x_{j}\right\|\right)
$$

where $H$ is Heaviside step function defined as

$$
H(x)=\left\{\begin{array}{lll}
0 & \text { when } & x \leq 0 \\
1 & \text { when } & x \geq 0
\end{array}\right.
$$

$N$ is the number of points in time series; $r$ is the radius of a sphere with its center at either of current points.

Then the correlation dimension is $D_{2}=$ $\lim _{r \rightarrow 0}(\log C(r) / \log r)$.

For the random process, $D_{2}$ varies linearly with the dimensions increasing without reaching a saturation value. If the system is chaotic, the slope of $\log C(r)$ versus $\log r$ converges to $D_{2}$ over an appropriate interval as $m$ increase.

3.3. Largest Lyapunov Exponent (LLE). The basic characteristics of chaotic dynamics are that the movement is extremely sensitive to initial conditions, two very close initial values resulting in orbit over time by separating exponentially, the Lyapunov exponent [21-23] describes the amount of this phenomenon.
Suppose that chaotic time series is $\left\{x_{1}, x_{2}, \ldots, x_{n}\right\}$, embedding dimension is $m$, and time delay is $\tau$; then reconstruction phase space is as follows:

$$
\begin{aligned}
& Y\left(t_{i}\right)=\left(x\left(t_{i}\right), x\left(t_{i+\tau}\right), \ldots, x\left(t_{i}+(m-1) \tau\right)\right), \\
& i=1,2, \ldots, N-(m-1) \tau .
\end{aligned}
$$

Take the initial point of phase space as $Y\left(t_{0}\right)$; suppose its most neighboring point is $Y_{0}\left(t_{0}\right)$; then the distance between these two points is $L\left(t_{0}\right)$. From time $t_{0}$ start tracking the time evolution of these two points, until the spacing between the two points exceeds a predetermined value $\varepsilon$ on the moment $t_{1}$ :

$$
L^{\prime}\left(t_{1}\right)=\left|Y\left(t_{1}\right)-Y_{0}\left(t_{1}\right)\right|>\varepsilon, \quad \varepsilon>0 .
$$

Retain point $Y\left(t_{1}\right)$; suppose that its most neighboring point is $Y_{1}\left(t_{1}\right)$; then

$$
L\left(t_{1}\right)=\left|Y\left(t_{1}\right)-Y_{1}\left(t_{1}\right)\right|<\varepsilon, \quad \varepsilon>0 .
$$

And make the angle $\theta$ between $L\left(t_{1}\right)$ and $L^{\prime}\left(t_{1}\right)$ as small as possible; repeat the above procedure until $Y(t)$ reaches the end of the time series; the total number of iterations is $M$; then the Largest Lyapunov exponent will be defined as follows:

$$
\lambda_{1}=\frac{1}{t_{M}-t_{0}} \sum_{k=1}^{M} \ln \frac{L^{\prime}\left(t_{k}\right)}{L\left(t_{k-1}\right)} .
$$

\section{Results}

The first minimum of the mutual information calculated for the lateral vibration and longitudinal vibration time series is obtained at $\tau=172$ (Figure 3(a)) and $\tau=160$ 


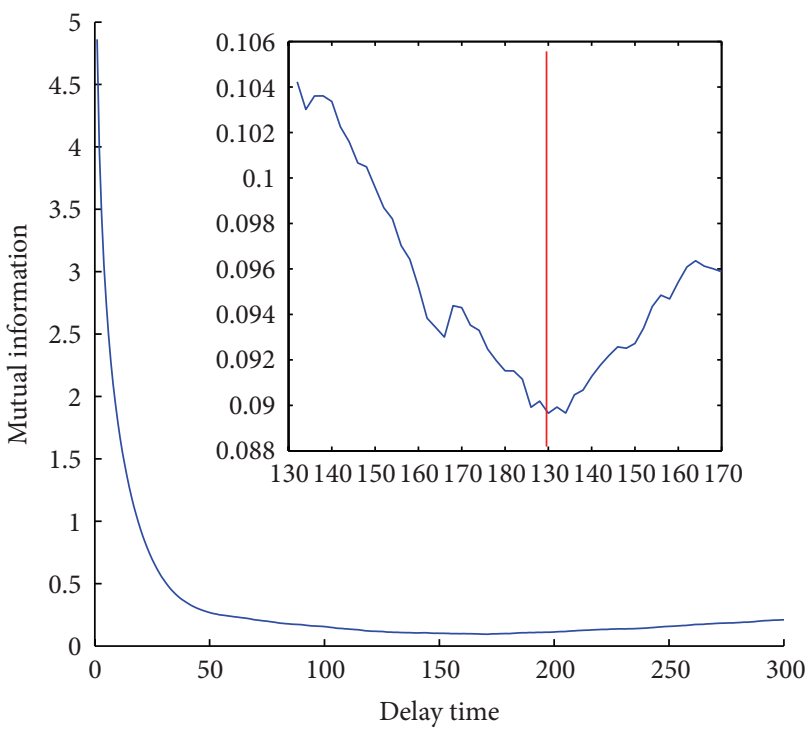

(a)

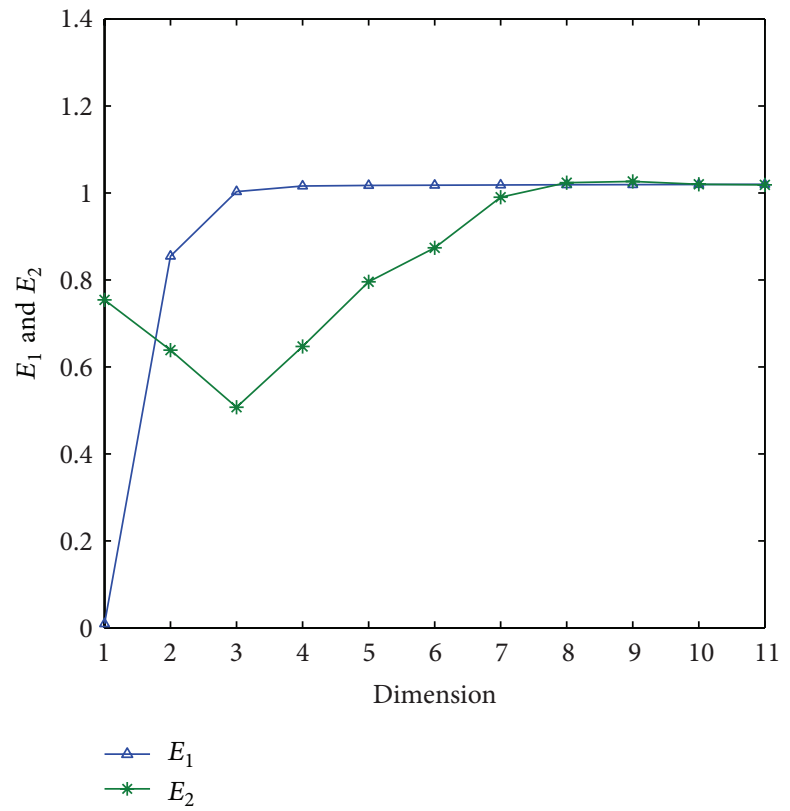

(b)

FIgURE 4: Determination of the proper delay time and embedding dimension of the longitudinal vibration $(\tau=160, d=8)$.

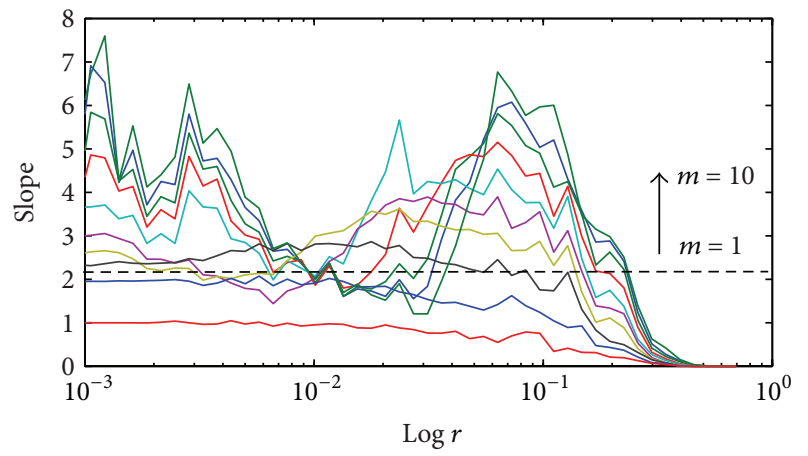

FIGURE 5: Local slope of correlation sum by lateral vibration time series.

(Figure 4(a)), which is also the value we will use in all subsequent calculations.

Calculate both $E_{1}(d)$ and $E_{2}(d)$ for determining the minimum embedding dimension of the time series and to distinguish deterministic data from random data. Shown in Figure 3 and Figure 4(b) are our results. Very clearly the minimum embedding dimension is 8 for both lateral and longitudinal vibration. Having calculated the optimal $\tau$ and $m$, we can successfully reconstruct the phase space of the system. The correct phase space reconstruction is a key step that enables further analyses of the dynamics associated with the drilling bit vibration.

The signals, which have chaotic characteristic, usually look like random, especially the nonlinear invariants which are similar to the noise. We are not fully aware of the nature of the drill string vibration signals performed as time series;

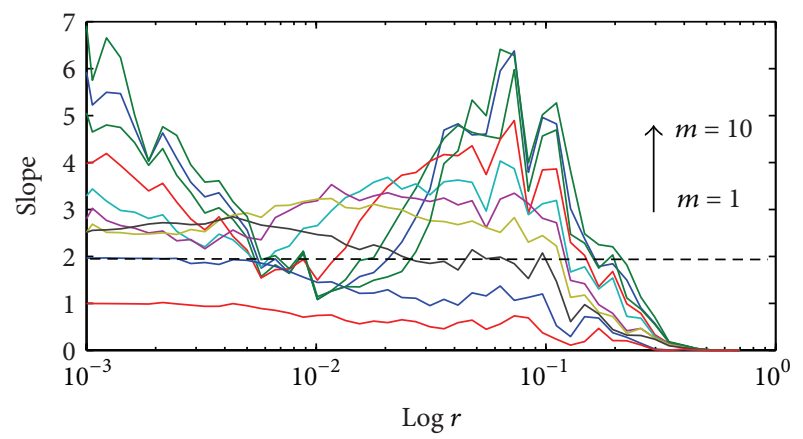

FIGURE 6: Local slope of correlation sum by longitudinal vibration time series.

the fluctuation of parameter $E_{2}$ in the Cao's method can be used as a measurement of uncertainty. When the system is chaos, $E_{2}$ will be gradually increased to one from zero as the embedding dimension increases. When the randomicity of the sequences enhancement, the fluctuation range of $E_{2}$ will become smaller and smaller; finally, $E_{2}$ will be approach to constant when the system is completely random, since there is no correlation between the data sets.

We use the Grassberger-Procaccia (GP) [19] algorithm which gives an estimate of the correlation dimension $D_{2}$. The results were displayed in Figure 5 and Figure 6. We present the estimated slope as a function of $\log r$ for embedding dimensions $m=1$ up to 10 , where we see that in the scaling region $\log r$ maybe have a region of convergence in the interval $[-2.4,-1.8]$ by the lateral vibration time series and in the interval $[-2.5,-2.0]$ by the longitudinal vibration 


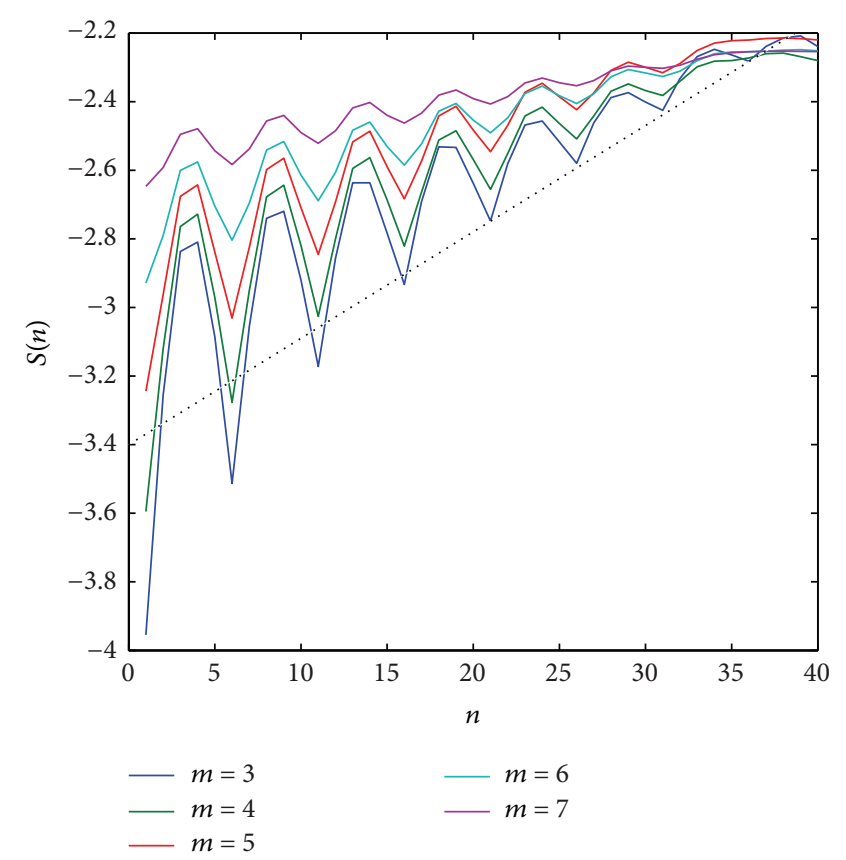

(a) Lateral vibration

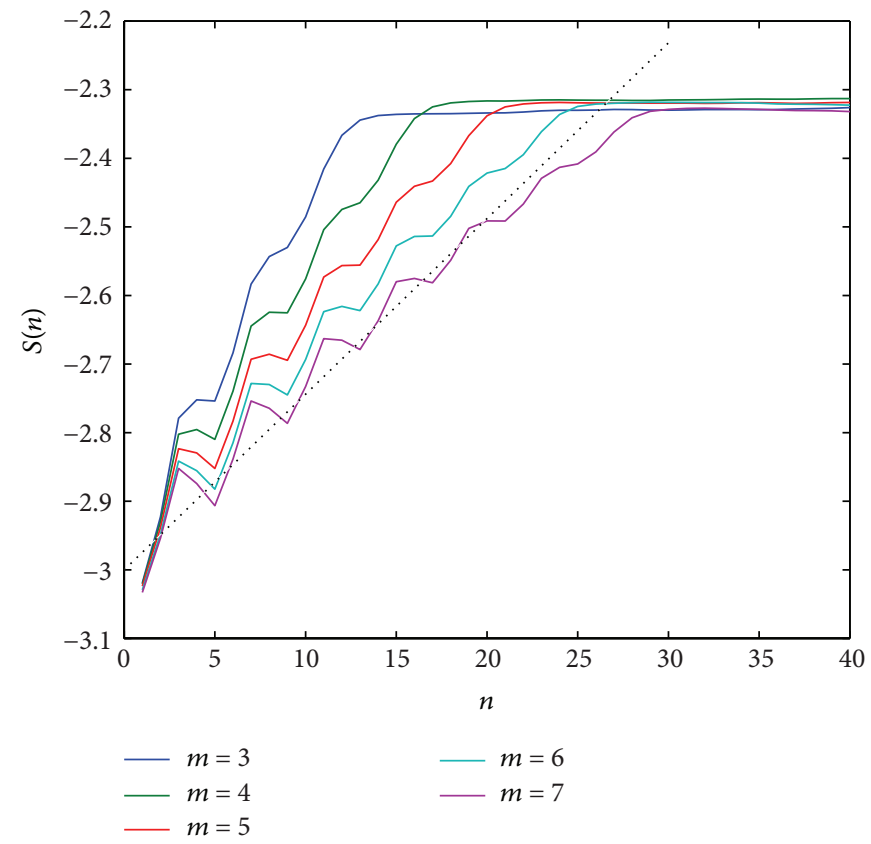

(b) Longitudinal vibration

FIGURE 7: Calculation of the largest Lyapunov exponent of the lateral vibration and longitudinal vibration and the effective expansion rate $S(n)$, of which the linear slope in dependence on $n$ is a robust estimate for the Largest Lyapunov exponent (using Tisean package [1], version 3.01).

time series. We can estimate the correlation dimension of lateral vibration in the range 2.0-2.5. This behavior indicates the possible existence of low-dimensional attractor with an estimate as $D_{2}=2.2 \pm 0.2$. We can also estimate the correlation dimension of longitudinal vibration in the range 2.0-2.2. This behavior indicates the possible existence of lowdimensional attractor with an estimate as $D_{2}=2.0 \pm 0.2$. For noise driven systems two points will be correlated only if they are close in time. In contrast, for chaotic systems, a larger correlation exists between points as the system is driven by a low-dimensional attractor, and order will be found in the random-like dynamic.

Next, we will calculate the largest Lyapunov exponent, which is one of the dynamic invariants to detect the existence of chaotic phenomena in the time series. We use the algorithm of Rosenstein et al. [22, 23] to calculate the LLE. The results were carried out with Tisean package [1], version 3.01. Consider the representation of the time series data as a trajectory in the embedding space, and assume that the point $S_{n}$ has a very closed neighbor point $S_{n^{\prime}}$. Then consider the distance $\Delta_{0}=S_{n}-S_{n^{\prime}}$ as a small perturbation, $\Delta_{l}=$ $S_{n+l}-S_{n^{\prime}+l}$. If one finds that $\left|\Delta_{l}\right| \approx \Delta_{0} e_{\lambda l}$, then $\lambda$ is the largest Lyapunov exponent. Assuming that $S(n)$ exhibits a linear increase with identical slope for all $m$ larger than some $m_{0}$ and for a reasonable range of $\varepsilon$, then this slope can be taken as an estimate of the largest exponent [1], $S(n)=$ $\left\{\ln \left(1 / u_{n} \sum_{s_{n^{\prime}} \in u_{n}}\left|s_{n+t}-s_{n^{\prime}+t}\right|\right)\right\}$.

The results obtained for $\varepsilon=0.02$ and $m=3-7$ are presented in Figure 7 . The function $S(n)$ shows a rather robust linear increase for all the embedding dimensions. Thus, the slope of $S(n)$, indicated by the dashed straight line, is a good estimate for the largest Lyapunov exponent of the system. The largest Lyapunov exponent of the lateral vibration is calculated through least-squares line fit for the time series and is found to be around 0.032. The largest Lyapunov exponent of the longitudinal vibration is calculated to be around 0.028 . This positive value indicates exponential divergence of trajectories and hence an evidence of chaos.

From the above, the results show that such system can exhibit positive finite-time Lyapunov exponents and a clear convergence toward the correlation dimension. Thus there is existence of low-dimensional chaotic in the drilling bit vibration, even though the data pollution was not removed completely and the saturation is not completely satisfactory.

\section{Conclusion and Discussion}

Drilling string vibration is a very common phenomenon that has attracted great interest of researchers. However, direct evidence of deterministic chaos of drill string vibration in practice is missing. Creating a more accurate theoretical model of the drill string vibration is difficult due to the impact of complex downhole conditions; it becomes possible to study the dynamic characteristics of drill string by measuring the vibration signal with the development of measurement technology. This paper found the existence of the deterministic chaotic characteristics of drill string vibration by time series analysis. The deterministic chaos has been studied 
overthrowing two centuries in a wide variety of experimental systems. Many ordered and disordered system behaviours have been interpreted and controlled using chaos theory.

Nowadays, the downhole drilling tool can achieve threedimensional vibration measurements with the development of downhole measurement technology, thus providing convincing data support for the studies of bottom drilling tool dynamics. Some even suggested the use of the downhole vibration signals in real time to determine formation lithology; however, these data are generally coming from some commercial companies, since the commercial competition, many researchers cannot get the real measurement data from the field test; many studies are also only limited to the laboratory. The data in this paper collected during hundreds of hours of drilling will be a time series with massive data. In order to reduce the amount of calculation, a typical section of time series is selected to be research object. Take a long segment of data to conduct an overall analysis of the drilling process. We use largest Lyapunov exponent as observed parameters to analyze the low-dimensional chaos during the entire drilling process. The results show that the chaotic phenomenon of the drilling bit vibration exists in the whole drilling process. In field measurement data of drill string vibration, finding chaotic characteristics is helpful to rediscover the drill string vibration phenomenon and to improve the control algorithm of the drill string vibration in engineering.

\section{Conflict of Interests}

The authors declare that there is no conflict of interests regarding the publication of this paper.

\section{Acknowledgments}

The authors express their appreciation to Drilling Technology Research Institute, Shengli Petroleum Administration of Sinopec Corp., for providing materials and allowing the publication of this paper. Also, thanks are due to the " 863 " project "Strap down Automatic Vertical Drilling System Engineering R\&D" (2009AA093501); "the Fundamental Research Funds for the Central Universities"(11CX06024A); "China Scholarship Fund" for their support.

\section{References}

[1] R. Hegger, H. Kantz, and T. Schreiber, "Practical implementation of nonlinear time series methods: the TISEAN package," Chaos, vol. 9, no. 2, pp. 413-435, 1999.

[2] W. C. Chin, Wave Propagation in Petroleum Engineering: Modern Applications to Drillstring Vibrations, MeasurementWhile-Drilling, Swab-Surge, and Geophysics, Gulf Publication, Houston, Tex, USA, 1994.

[3] S. L. Chen and M. Géradin, "An improved transfer matrix technique as applied to BHA lateral vibration analysis," Journal of Sound and Vibration, vol. 185, no. 1, pp. 93-106, 1995.

[4] A. P. Christoforou and A. S. Yigit, "Dynamic modelling of rotating drillstrings with borehole interactions," Journal of Sound and Vibration, vol. 206, no. 2, pp. 243-260, 1997.
[5] R. D. Graham, M. A. Frost III, and J. C. Wilhoit Jr., "Analysis of the motion of deep-water drill strings-part 1: forced lateral motion," Journal of Engineering for Industry, vol. 87, no. 2, pp. 137-144, 1965.

[6] R. Plunkett, "Static bending stresses in catenaries and drill strings," Journal of Engineering for Industry, vol. 89, no. 1, pp. 31-36, 1967.

[7] M. A. Vaz and M. H. Patel, "Analysis of drill strings in vertical and deviated holes using the Galerkin technique," Engineering Structures, vol. 17, no. 6, pp. 437-442, 1995.

[8] A. S. Yigit and A. P. Christoforou, "Coupled torsional and bending vibrations of drillstrings subject to impact with friction," Journal of Sound and Vibration, vol. 215, no. 1, pp. 167-181, 1998.

[9] P. D. Spanos, A. M. Chevallier, and N. P. Politis, "Nonlinear stochastic drill-string vibrations," Journal of Vibration and Acoustics, vol. 124, no. 4, pp. 512-518, 2002.

[10] H. Chunjie and Y. Tie, "The research on axial vibration of drill string with Delphi," in Proceedings of the International Conference on Computational Intelligence and Natural Computing (CINC '09), pp. 478-481, Wuhan, China, June 2009.

[11] T. Richard, E. Detournay, M. Fear, B. Miller, R. Clayton, and O. Matthews, "Influence of bit-rock interaction on stick-slip vibrations of PDC bits," in Proceedings of the 2002 SPE Annual Technical Conference and Exhibition, MS77616, pp. 2407-2418, San Antonio, Tex, USA, September 2002.

[12] K. Mongkolcheep, A. Palazzolo, A. Ruimi, and R. Tucker, "A modal approach for chaotic vibrations of a drillstring," in Proceedings of the ASME International Design Engineering Technical Conferences and Computers and Information in Engineering Conference (DETC '09), pp. 1305-1314, San Diego, Calif, USA, August 2009.

[13] A. D. Craig, R. Goodship, and D. R. Shearer, "High frequency downhole dynamic measurements provide greater understanding of drilling vibration in performance motor assemblies," in Proceedings of the 2010 IADC/SPE Drilling Conference and Exhibition, pp. 377-386, New Orleans, La, USA, February 2010.

[14] L. W. Ledgerwood III, O. J. Hoffmann, J. R. Jain, C. El Hakam, C. Herbig, and R. W. Spencer, "Downhole vibration measurement, monitoring and modeling reveal stick-slip as a primary cause of PDC bit damage in today's applications," in Proceedings of the 2010 SPE Annual Technical Conference and Exhibition, pp. 26522661, Florence, Italy, September 2010.

[15] P. D. Welch, "The use of fast Fourier transform for the estimation of power spectra: a method based on time averaging over short, modified periodograms," IEEE Transactions on Audio Electroacoustics, vol. 15, no. 62, pp. 70-73, 1967.

[16] A. M. Fraser and H. L. Swinney, "Independent coordinates for strange attractors from mutual information," Physical Review A, vol. 33, no. 2, pp. 1134-1140, 1986.

[17] L. Cao, "Practical method for determining the minimum embedding dimension of a scalar time series," Physica D, vol. 110, no. 1-2, pp. 43-50, 1997.

[18] M. B. Kennel, R. Brown, and H. D. I. Abarbanel, "Determining embedding dimension for phase-space reconstruction using a geometrical construction," Physical Review A, vol. 45, no. 6, pp. 3403-3411, 1992.

[19] P. Grassberger and I. Procaccia, "Measuring the strangeness of strange attractors," Physica D, vol. 9, no. 1-2, pp. 189-208, 1983.

[20] P. Grassberger and I. Procaccia, "Estimation of the Kolmogorov entropy from a chaotic signal," Physical Review A, vol. 28, no. 4, pp. 2591-2593, 1983. 
[21] A. Wolf, J. B. Swift, H. L. Swinney, and J. A. Vastano, "Determining Lyapunov exponents from a time series," Physica D, vol. 16, no. 3, pp. 285-317, 1985.

[22] M. T. Rosenstein, J. J. Collins, and C. J. de Luca, "A practical method for calculating largest Lyapunov exponents from small data sets," Physica D, vol. 65, no. 1-2, pp. 117-134, 1993.

[23] H. Kantz, "A robust method to estimate the maximal Lyapunov exponent of a time series," Physics Letters A, vol. 185, no. 1, pp. 77-87, 1994. 

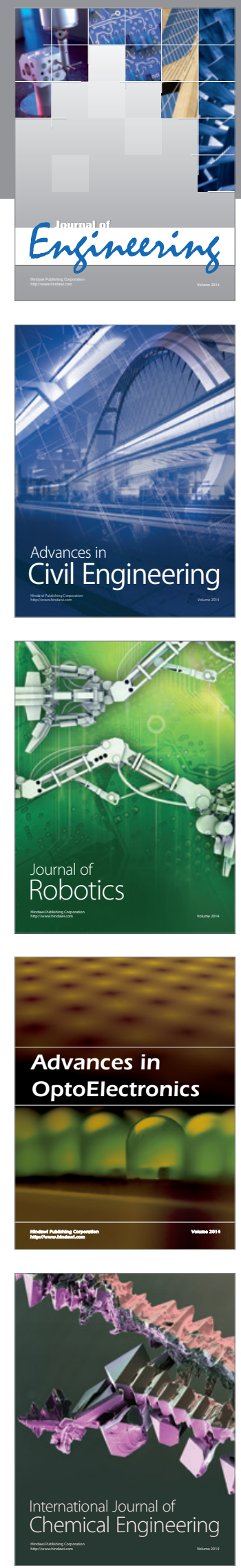

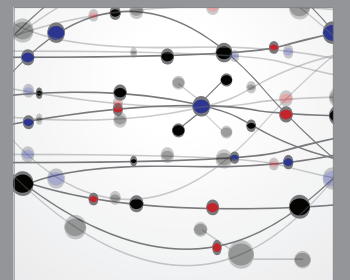

The Scientific World Journal
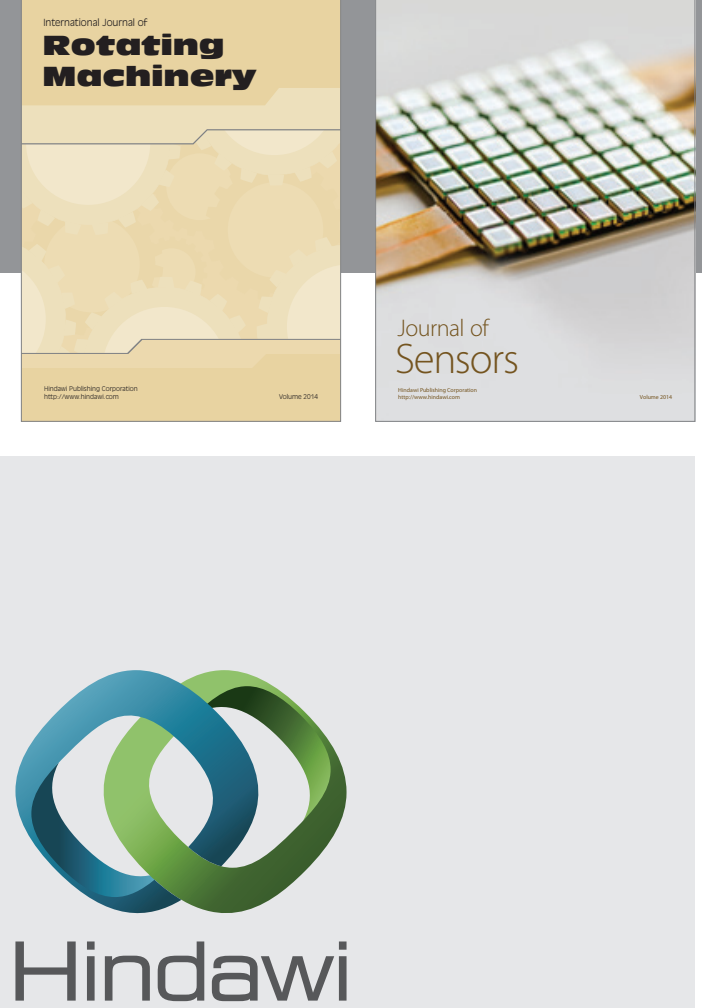

Submit your manuscripts at http://www.hindawi.com
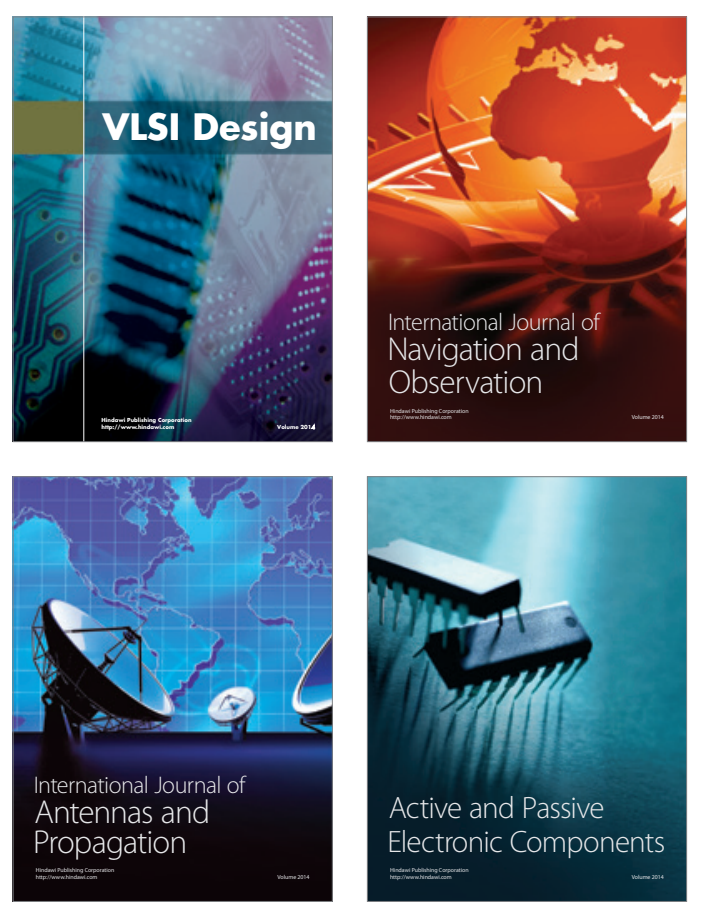
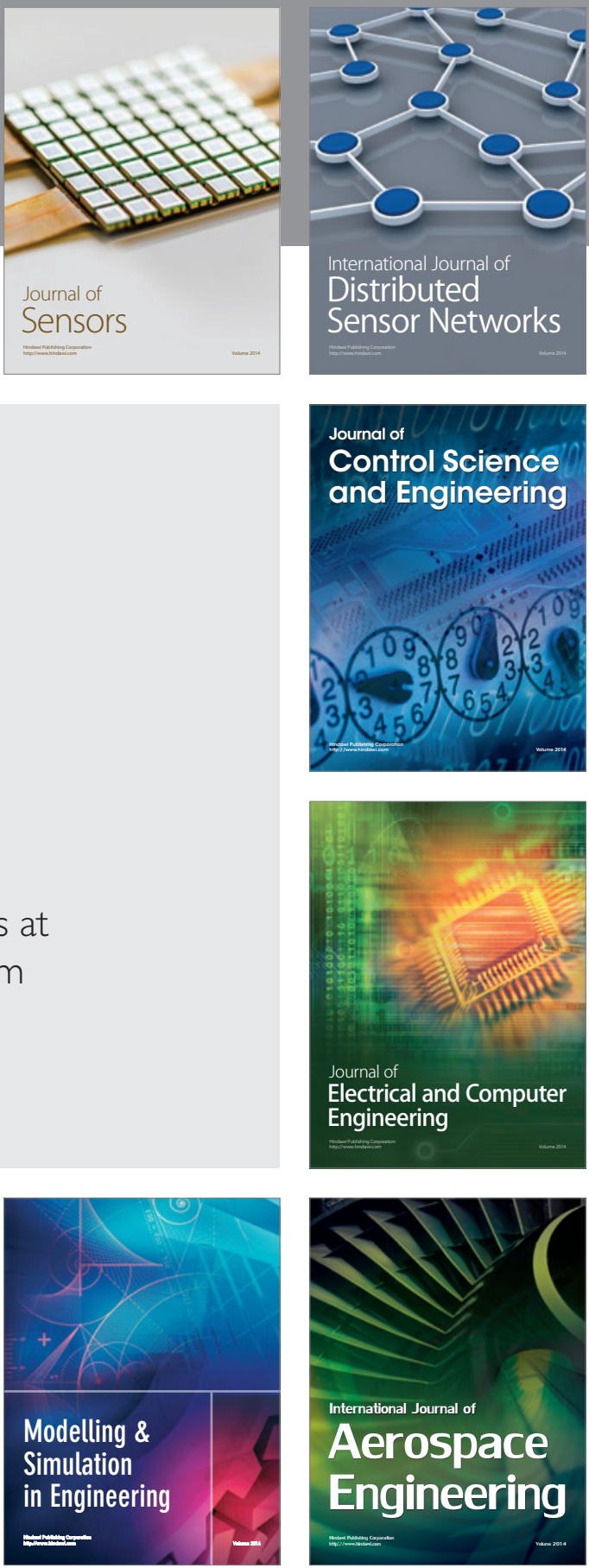

Journal of

Control Science

and Engineering
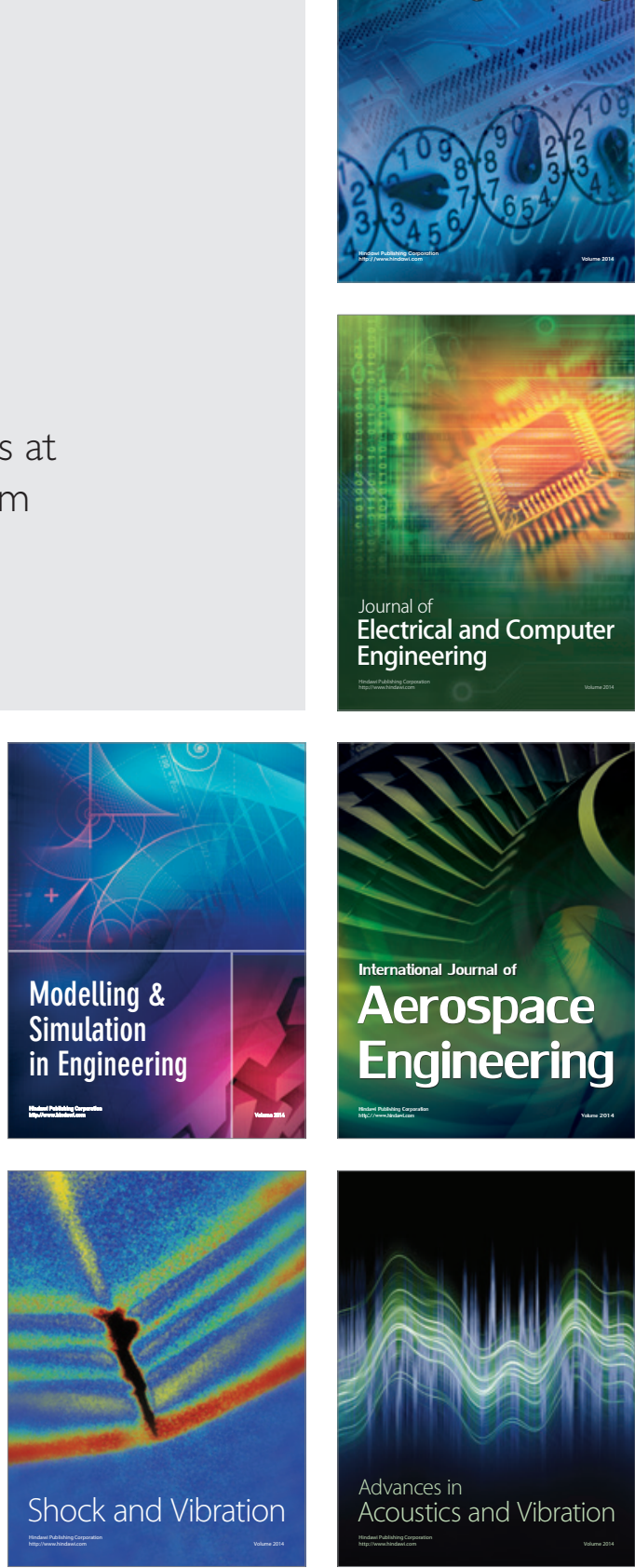